\title{
Evolution and entropy in the organization of urban street patterns
}

\author{
Nahid Mohajeri ${ }^{\mathrm{a} *}$, Jon R. French ${ }^{\mathrm{b}}$ and Michael Batty ${ }^{\mathrm{c}}$ \\ ${ }^{a}$ Department of Geography, University College London, London, UK; ${ }^{b}$ Environmental Modelling Group, Department of Geography, \\ University College London, London, UK; ${ }^{c}$ Centre for Advanced Spatial Analysis (CASA), University College London, London, UK
}

(Received 1 December 2012; final version received 10 December 2012)

\begin{abstract}
The street patterns of cities are the result of long-term evolution and interaction between various internal, social and economic, and external, environmental and landscape, processes and factors. In this article, we use entropy as a measure of dispersion to study the effects of landscapes on the evolution and associated street patterns of two cities: Dundee in Eastern Scotland and Khorramabad in Western Iran, cities which have strong similarities in terms of the size of their street systems and populations but considerable differences in terms of their evolution within the landscape. Landscape features have strong effects on the city shape and street patterns of Dundee, which is primarily a shoreline city, while Khorramabad is primarily located within mountainous and valley terrain. We show how cumulative distributions of street lengths when graphed as $\log -\log$ plots show abrupt changes in their straight-line slopes at lengths of about $120 \mathrm{~m}$, indicating a change in street functionality across scale: streets shorter than $120 \mathrm{~m}$ are primarily local streets, whereas longer streets are mainly collectors and arterials. The entropy of a street-length population varies positively over its average length and length range which is the difference between the longest and the shortest streets in a population. Similarly, the entropies of the power law tails of the street populations of both cities have increased during their growth, indicating that the distribution of street lengths has gradually become more dispersed as these cities have expanded.
\end{abstract}

Keywords: city growth; urban morphology; street patterns; size distribution; entropy measures

\section{Introduction}

The dynamics of urban morphology has been explored from many different perspectives (e.g. Harris 1985; Berechman and Small 1988; Yongmei and Junmei 2004; Benguigu, Blumenfeld-Lieberthal, and Czamanski 2006; Batty 2008, 2010), but for a better understanding of city structure and the complexities of this dynamics, it is crucial to quantify the different physical properties of those structures. To advance this, we argue here that street networks are among the most important city structures, and recently, there have been many studies of street patterns and city growth based on socio-economic data (Batty 1971; Wegener 1994; Makse, Havlin, and Stanley 1995; Hillier 1999; Barredo Kasanko, McCormick, and Lavalle 2002; Berling and $\mathrm{Wu}$ 2004). There have been also been many analyses of street patterns based on network science which, in the last decade, has become highly significant (Cardillo et al. 2006; Scellato et al. 2006; Jiang 2007; Barthelemy and Flammini 2008; Masucci et al. 2009) while related studies such as those by Xie and Levinson (2007), Lammer et al. (2006), Jiang (2009) and Levinson and Huang (2012) amongst others focus on the structural properties of road networks from the point of view of traffic and engineering. Furthermore, Marshall (2005) explains how different layouts and patterns of streets contribute to better urban design, addressing how design aspects of urban transportation might increase the functionality of cities.

By contrast, research on street patterns and urban dynamics based on physical data and how they relate to physical concepts is much less developed and remains in its infancy. In particular, with few exceptions (Mohajeri 2012; Mohajeri and Gudmundsson 2012), there has been little attempt to analyse city growth and street patterns in relation to landscape using entropy concepts. There is a clear need for rigorous quantitative methods that explain (1) how city geometry changes over time as a function of its size and of external landscape constraints and (2) how changes in city geometry affect the associated street patterns that determine how energy is distributed within the city in terms of the flow of people and materials. One rigorous quantitative method which has found extensive use in systems theory and indeed in spatial interaction modelling is entropy analysis. Entropy statistics, which measures the variation of a phenomenon with respect to its frequency across a given

\footnotetext{
*Corresponding author. Email: mohajeri.nahid.09@ucl.ac.uk
} 
range, allows us to quantify changes in geometry as a city grows and helps in assessing the plausible mechanisms for the formation and evolution of city structure.

In this article, our main aim is to use entropy analysis to show how the properties of street patterns, focusing on street lengths, vary within a city and how this variation partly reflects external landscape constraints. The second aim is to investigate the degree to which landscape constraints, such as coastlines, mountain ranges and major rivers, control the shape of cities by providing constraints on their growth. This article is empirically grounded in that it focuses on the associations between landscape, city evolution and street patterns of two case studies, namely the cities of Dundee in Scotland and Khorramabad in Iran (Figures 1-2).

\section{Case study exemplars: the geographical background}

We need to first justify the selection of these two cities which are quite different in terms of both their physical and cultural contexts. Both cities have clear boundaries. Their overall shape is partly controlled by their landscape, primarily the coastline of the Firth of Tay in the case of Dundee (Figure 1) and mountains and valleys in the case of Khorramabad (Figure 2). This is somewhat different from cities, such as Paris or Chicago, whose landscape morphologies do not have such strong physical features. The availability of historical data and GIS data sets for street networks of both cities also makes it possible to carry out a detailed analysis of their street networks.

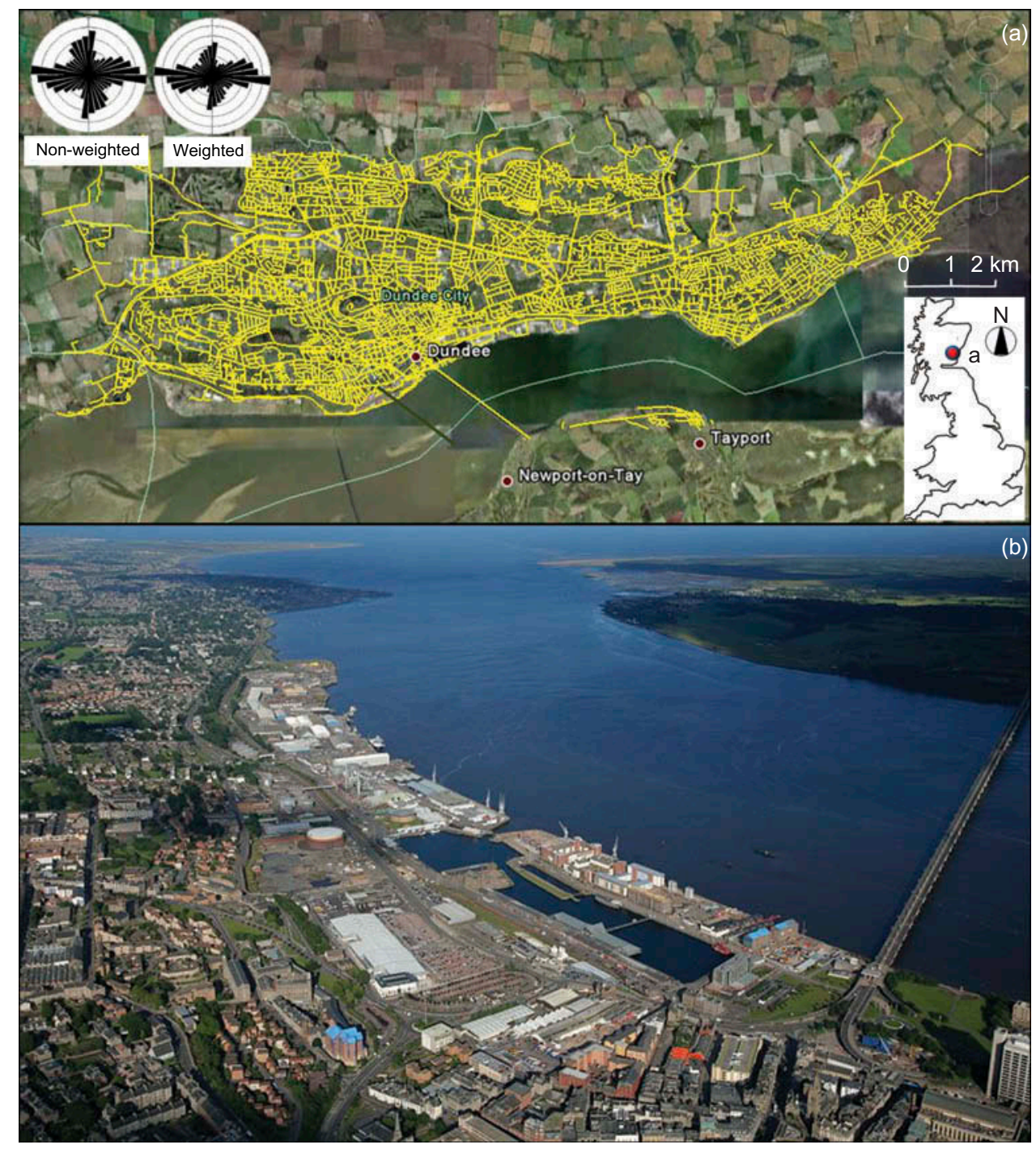

Figure 1. (a) Location of the city of Dundee, Eastern Scotland (image courtesy of Google Earth), with street patterns overlaid. (b) Aerial view of the Dundee Docks with the Dock Street running diagonally across the centre of the picture (Guthrie aerial photography).

Notes: The rose diagrams (top-left) summarise the weighted and non-weighted trend distributions of all the streets $(N=9616)$. Each rose presents the trend with a 10 degree interval and 0-360 degree azimuth (modified from Mohajeri and Gudmundsson 2012). 

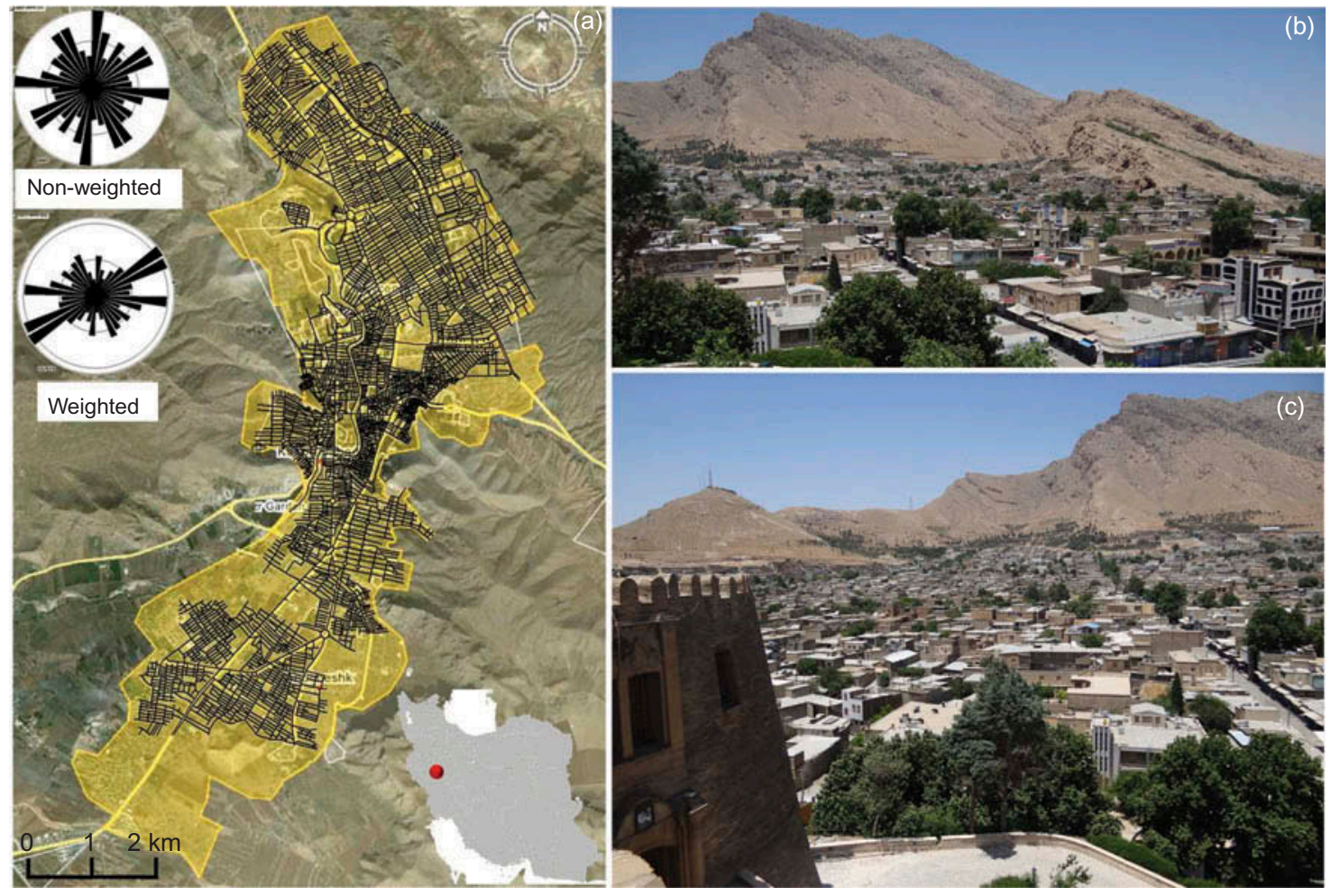

Figure 2. (a) The city of Khorramabad in Western Iran. (b and c) Aerial views of the city of Khorramabad (Photo by Adam Jones, Ph.D./Global Photo Archive).

Notes: Its geometry is largely controlled by landscape constraints, primarily mountain slopes or fronts and valleys. The two rose diagrams summarise the general trend of all the 8481 streets using weighted and non-weighted data.

An additional set of reasons is the striking difference in their history of evolution. Dundee has been developing gradually over several hundred years; its inner part dates back to the medieval times and is thus historically important (Ferguson 2005; Watson 2006). The city, in fact, dates back to at least to the twelfth century and has a current population of 143,390 (General Register Office for Scotland 2009). It is located along the north coast of a fjord, the Firth of Tay Estuary, in Eastern Scotland (Figure 1) and is Scotland's fourth largest city. The city has a roughly elliptical boundary, part of which is determined by the shoreline of the Tay.

By contrast, the greater part of Khorramabad, in Western Iran, is a very young city, mostly less than 60 years old. The city population is about 334,000 (Iranian Statistical Centre 2007). It is located in the province of Lorestan and is surrounded by prominent landform features such as mountainscapes that form part of the Zagros range (Figure 2). It thus provides an excellent example of a rapidly expanding city subject to strong landscape constraints. There exist detailed maps of the city since 1955 , at which time it occupied only the small, narrow (bottleneck) part of the present city (Figure 2). The south and southeast and north and northwest parts of the city extend to form 'wings' within its valley. The wings are connected by a narrower pass through the mountains, which functions as a natural bottleneck that constrains traffic between the two parts of the city. At its narrowest, the width of this bottleneck is only $1.1 \mathrm{~km}$. The overall shape of Khorramabad is broadly that of a crescent, with somewhat irregular boundaries that are clearly constrained by the valley of the same name, its flanking mountains and the narrow pass.

\section{Statistical methods}

\subsection{Data sources}

Transport network data sets for the United Kingdom were available from the Integrated Transport Network (ITN) layer (provided by the Ordnance Survey), downloadable from the UK EDINA Digimap website (Digimap: http:// www.edina.ac.uk/). This layer consists of the road network, road routing information and other transport information. Street data sets and their statistical information within the city for Dundee were obtained from the Digimap source and imported into GIS (Arcview Version 9.3; www.esri.com) while then the ITN layers were converted to GIS-shapefile format. Dundee City Council and the National Library of Scotland also provided the historical data for previous street networks in Dundee, and all 
the historical maps were digitised for import into ArcGIS. The National Iranian Cartographic Centre (2005) provided the GIS-shapefiles for the network data sets of the city of Khorramabad. In addition, the CAD/GIS master plan of the city is used so as to obtain the most recent street networks of city (Ministry of Housing and Urban Development 2005). The historical maps of Khorramabad were digitised through GIS, the maps being scanned from master plan studies of the city (Ministry of Housing and Urban Development 2005). Google Earth was also used for capturing appropriate remote-sensing images of two cities which display the main physical features and act as a backcloth to the street line analysis. As our analysis will focus on the variation in street-segment lengths and trends (orientation or azimuth) and street spacing, a street segment is defined as the distance from one junction to the next while spacing is defined as the shortest distance between street centrelines. External constraints considered here include landscape factors such as coastlines (Dundee city) and mountain ranges (Khorramabad) as such constraints largely define the boundary shape of each of the case study cities. Digital terrain models were used to supplement such as those used in Google Earth.

\subsection{Analytical methods}

\subsubsection{Delineation of city boundaries}

There is no definitive agreement on how to define a city boundary and methods vary depending on the application (Benguigui, Blumenfeld-Lieberthal, and Czamanski 2006; Pont and Haupt 2010). Here the boundaries are determined from aerial imagery (Google Earth and various aerial photographs) on the basis of changes in land cover and clear geomorphological features. In the case of Dundee, the estuary shoreline forms a natural boundary to the south and southeast, and in the north and northwest, there is a very clear transition from urban to agricultural land use. In the case of Khorramabad, the agricultural field patterns and the steep and sharp mountain slopes clearly separate both cities from their surroundings and allow clear delineation of boundary polygons.

\subsubsection{Directional statistics}

The distribution of street orientations is presented using rose diagrams (Swan and Sandilands 1995; Smith, Goodchild, and Longley 2009), constructed using the program GEOrient (http://www.geoorient.com/). Two sets of analyses were performed; first, using non-normalised (nonweighted) data, where short streets and long streets have equal weight in the rose diagram, and second, using data normalised (weighted) in proportion to the length of the shortest street. In this case, more weight is given to longer streets which are in proportion to their lengths.

\subsubsection{Power law size distributions}

Power law size distributions are very common in artificial (man-made) and natural processes and structures, particularly in the heavy tails of many distributions which often account for the majority of size or volume of the range of objects in question. The populations of cities, the intensities of earthquakes, word frequencies in literature and the frequencies of family names all give rise to power law-like distributions (e.g. Schroeder 1991; Peitgen, Jurgens, and Saupe 2004; Newman 2005). Skew distributions in general and power-law distributions in particular imply that the number of small events, processes or objects of a particular type is large in comparison with the number of large events, processes or objects of the same type. In general, systems where competitive processes are at work usually determine this sorting of small from large which often accords to evolution where the dynamics of the system is key. When applied to a cumulative frequency (probability) distribution, a power law has the form:

$$
P(\geq x)=C x^{-D}
$$

where $P(\geq x)$ is the number of objects with a size larger than $x, C$ is a constant of proportionality and $D$ is the scaling exponent. In the case of a distribution of street lengths, $P(\geq x)$ is the number of streets with a length larger than $x, C$ is constant and $D$ is the scaling exponent. To determine whether data sets follow a power law distribution, the traditional and standard procedure is simply to plot the logarithms of the values $(x)$ and their probabilities $P(x)$ as $\log (P(x))=\log (C)-D \log (x)$. A straight line on the $\log -\log$ plot is then usually regarded as a general indication of that a power law can account for the variation (Newman 2005; Jiang 2007, 2009; Clauset, Shalizi, and Newman 2009) but in reality, however, a straight line is hardly ever observed over the entire range of the values or sizes of $x$; there is normally a cut-off at the smallest perceivable size (Newman 2005). Thus, the distribution generally corresponds to a power law only over a certain range, for example, in its heavy tail or short tail, dependent on what transform of the distributions is being examined. To validate size distributions as a power law, the maximum likelihood method generates the most acceptable statistics used to compare the power law fit with other candidates such as the log-normal, exponential and stretched exponential. Details of this estimation procedure are given in Section 5.

\subsubsection{Entropy}

Entropy, commonly denoted by the symbol $S$, is a fundamental thermodynamic concept. In classical thermodynamics, an infinitesimal entropy change, $d S$, is defined as: 


$$
d S \geq \frac{\delta Q}{T}
$$

where $\delta \mathrm{Q}$ is the energy (heat) received or absorbed by the system under consideration, and $T$ is the absolute (Kelvin) temperature (of the source) at the time when that energy/heat is received. The equality sign applies to reversible processes - the inequality sign to irreversible processes - and the units of $\delta S$ are given in energy (joules) over absolute temperature $(K)$, or $J K^{-1}$. A thermally isolated system cannot receive any heat from an environment, in which case $\delta Q=0$ and, from Equation (2), $d S \geq 0$ which may be regarded as one version of the second law of thermodynamics. It implies that for any change in such a system, its entropy either stays the same (a reversible change) or increases (an irreversible change). In cases where the system is not isolated, its entropy may decrease as it imports energy from it surrounding parts. However, the entropy of the system and its surroundings must increase if the systems and its surroundings are isolated, hence self-contained.

As defined above, this traditional variety of entropy does not have an immediate application to street patterns in terms of their evolution, at least as we have used it here but physical entropy also has a basis in probability theory through statistical mechanics. When related to a probability, the concept of entropy can be used in analysing the frequency distribution of streets using the following expression, known as Shannon-Gibb's entropy formula, which gives the entropy for a general probability distribution (Dill and Bromberg 2003; Blundell and Blundell 2006) as

$$
S=-k \sum_{i=1}^{t} P_{i} \ln P_{i}
$$

Where $k$ is a constant that is usually taken as the dimensionless number 1, when dealing with frequency distributions (Ben-Naim 2008; Volkenstein 2009). For a power law distribution of street lengths, $t$ is defined as the number of classes or bins that contains streets in the frequency distribution, that is, the number of bins of street lengths with nonzero probabilities of streets, and $P_{i}$ is the frequency or probability of a set of streets belonging to the $i$-th bin, that is, the probability of the $i$-th class or bin (Dill and Bromberg 2003; Volkenstein 2009). When calculating the entropy using Equation (3), it is usual to include only those bins where the probability of finding a street is greater than zero (thus, each included bin contains at least one street). Equation (3) is analogous to the Shannon entropy equation, which lies at the basis of information theory (Jaynes 1957) and is here applied to frequency distributions (Wang et al. 2003; Rao et al. 2004; Drissi, Chonavel, and Boucher 2008; Navarro, Aguila, and Asadi 2010; Chen 2012). By definition, we also have

$$
\sum_{i=1}^{t} P_{i}=1
$$

where the sum of the probabilities for all the bins is equals to one. Given that the probabilities are always between 0 and 1 (Equation (4)), and the natural logarithm of numbers between 0 and 1 is negative, the minus sign in Equation (3) ensures that entropy must always be positive. The probabilities, as applied to streets in a population, are a measure of the chances of randomly selected streets from the population of street lengths falling into a particular bin.

The calculated entropy of the population depends on the shape of the probability distribution. For example, if the distribution is uniform, that is, all the bins occupied by streets have the same lengths (heights), so that the probability of streets belonging to any of the bins is equal, then the entropy reaches its maximum value (Kondepudi and Prigogine 1998; Stamps 2004; Nelson 2006; Desurvire 2009; Volkenstein 2009). The entropy of an isolated system in a given macrostate where all the probabilities are equal may be derived from Equation (3) and is given by the Boltzmann equation, namely:

$$
S=-k \ln (1 / t)=k \ln t
$$

where, again, $t$ is the number of nonzero bins in the probability or frequency distribution.

A city, however, is not and cannot in any sense be treated as an isolated system since it always exchanges materials, energy, information and people with its surroundings. A street network as a part of a developing city is thus not isolated (it may be either closed or open). It thus follows that the bins or classes (microstates in statistical mechanics) for a street network are not equally probable. It is of interest to examine how this measure changes as a city develops, for it is a signature of how evenly spread are the distribution of street lengths and this reflects the extent to which the city is evolving and changing.

\section{Street patterns and size distribution}

\subsection{Dundee}

The trends for the whole city (with 9616 street segments, Figure 1a) and those within sub-regions along its estuarine shoreline (6004 street-segments, Figure 3) were analysed and presented as rose diagrams, using both normalised and non-normalised data. The sub-regions (Figure 3) were chosen according to three criteria, namely: (1) The number of streets should be similar in all the subareas (800<N<900); (2) All the subareas should be of a similar size; and (3) The subsets should reflect the variation in alignment of the shoreline.

From Figure 1, it is evident that there are two main street trends in Dundee: one aligned roughly northsouth, 


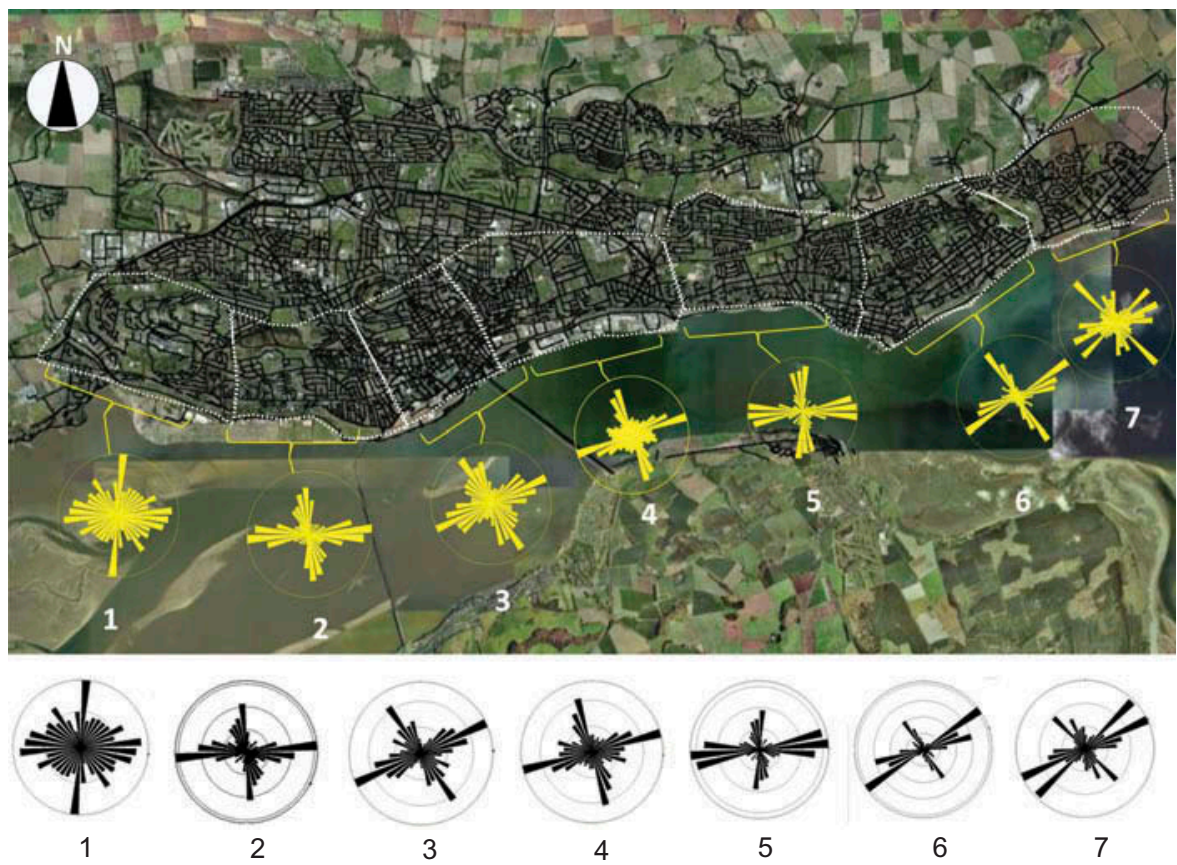

Figure 3. Variations in street trend for subareas of Dundee that lie along its estuarine shoreline.

Notes: Each rose (1-7) on the image presents the trend within a chosen part of the city with a 10 degree interval. The main, roughly orthogonal, street trends are coast-parallel and coast-perpendicular and follow variations in the trend of the coastline. On the image, the data are non-weighted, but below the image the data are weighted (modified from Mohajeri and Gudmundsson 2012).

the other roughly eastwest. These are broadly shorelineperpendicular and coast-parallel respectively. The extent to which the shore-parallel trends closely follow variation in shoreline direction is strikingly evident from Figure 3. It is notable that, progressing along the shoreline from west to east, the northerly trending streets remain orthogonal and thus become north-northwest trending towards the eastern part of the city. Greater variability in street direction at the eastern and, especially, at the western margins of the city are partly attributable to many streets being roughly perpendicular to the curved landward boundary of the city at these localities. The change in trend towards the city centre is presumably because this is the oldest part, where the city originated and where the segments tend to be more irregular (see the non-weighted rose 3 in Figure 3). Coast-parallel street segments tend to be longer than the coast-perpendicular segments. This is presumably because the city originated with the first harbour on the estuary shore and subsequently grew preferentially along this shoreline.

Cumulative distributions (Equation (1); Figure 4a) are used to explore the power law properties of street lengths. Log- $\log$ plots (Figure $4 \mathrm{~b}$ and c) suggest that the street length distributions are consistent with composite power laws that have different scaling exponents for different street-length ranges. From purely visual inspection, a clear break in straight-line slope occurs at a street length of around $140 \pm 20 \mathrm{~m}$, at which point the scaling exponent changes from 0.917 to 2.582 . This implies the existence of two distinct street populations. That composed of streets with lengths from $3 \mathrm{~m}$ to $140 \mathrm{~m}$ primarily consists of local streets, including private lanes and alleys and cul-desacs (Headicar 2009). The other population (streets with lengths from $140 \mathrm{~m}$ to $2248 \mathrm{~m}$ ) is comprised primarily of local roads and collectors (that are commonly wider than local roads and feed the traffic from local streets to arterial roads).

\subsection{Khorramabad}

The analysis of 8481 streets for Khorramabad again indicates the existence of two dominant trends (Figure 2b), although these are much weaker than in the previous case of Dundee. The greater variation in street orientation for Khorramabad is partly due to the broadly crescent-shaped city boundary, which is more directly constrained by landscape topography. To further explore this, the city was divided into five similarly sized (average $N=1692$ ) subareas (Figure 5) based on different time periods of the city growth. Weighted and non-weighted rose diagrams for these subareas exhibit more clearly bi-directional orientations that, for Dundee, are clearly aligned with constraining external boundary (the coastline).

Results for all the street lengths are shown in Figure 6, which provides cumulative plots of all the streets lengths exceeding a given length against the lengths 


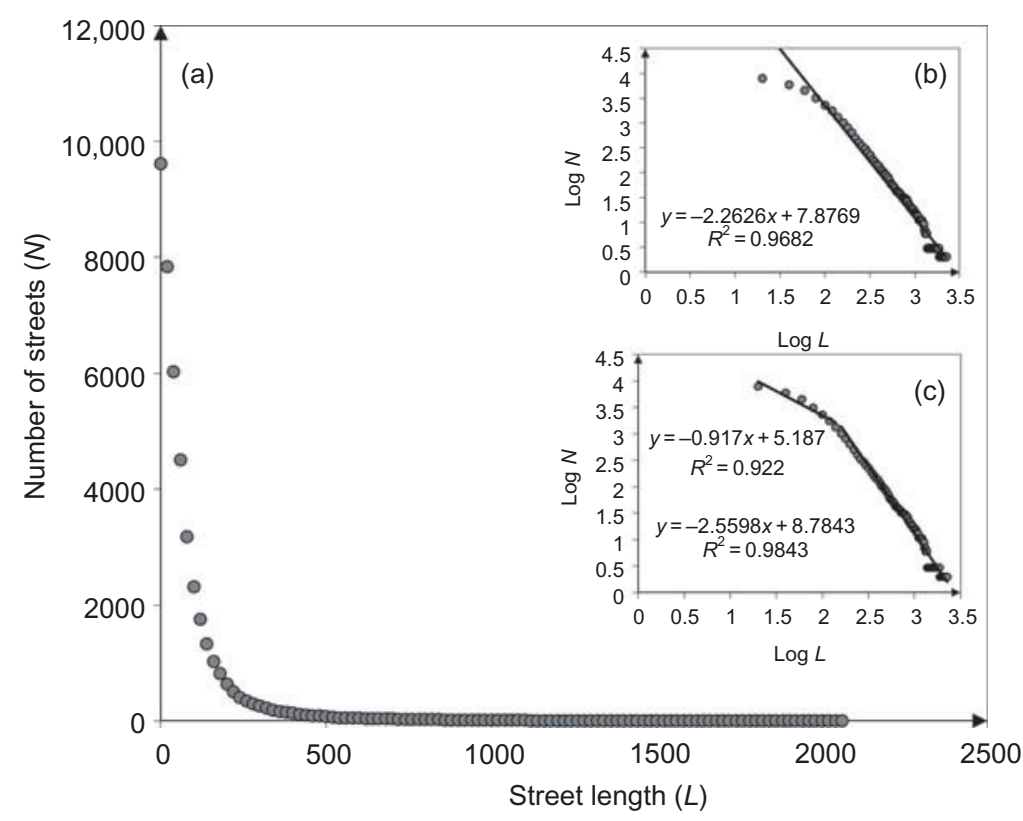

Figure 4. Power law frequency plot of all street segments (9616) measured in Dundee. (a) Ordinary cumulative length distribution (b) Single-line $\log -\log$ fit. (c) Double-line $\log -\log$ fit. The different scaling exponents indicate different street populations.

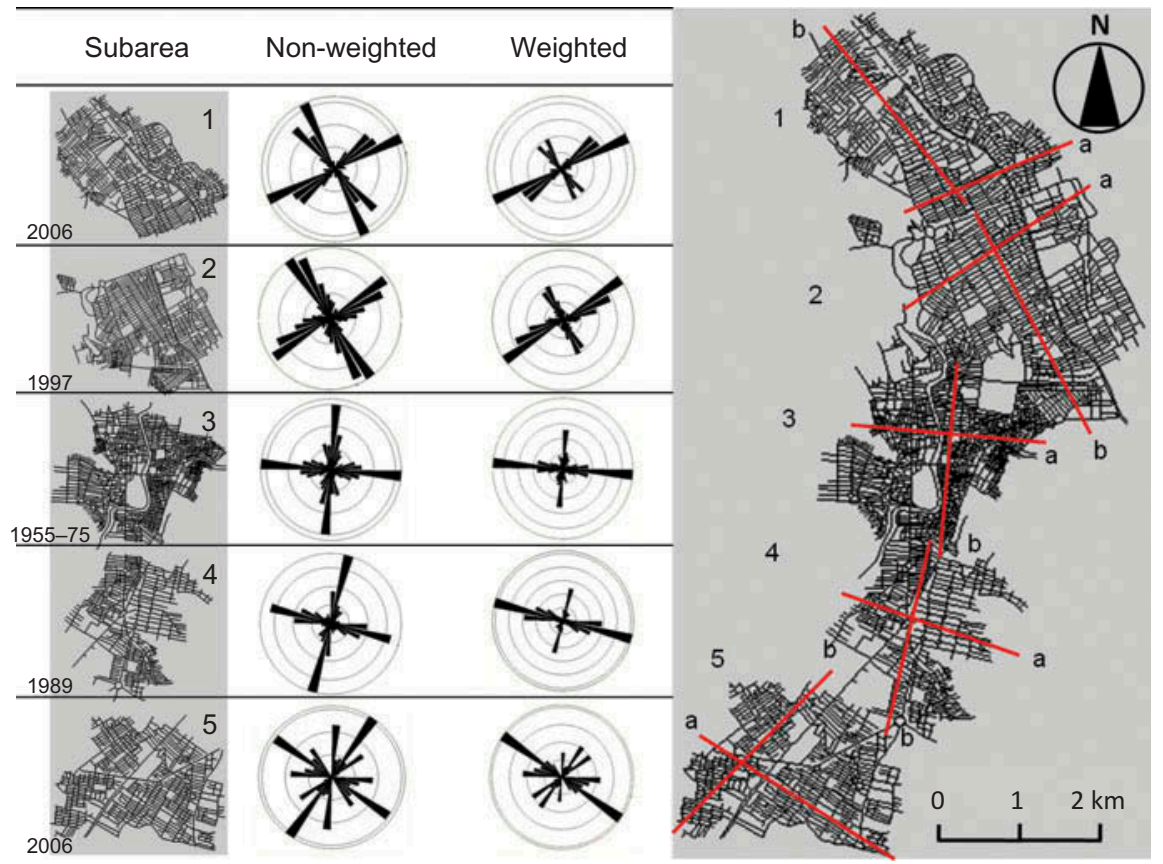

Figure 5. The city of Khorramabad (right): divided into five subareas/subsets, each with a subpopulation of streets (numbered 1-5).

Notes: The roughly easterly dominating main trend is perpendicular to the curved boundaries of the crescent-shaped city. The main, roughly northerly, subordinate trend is parallel with the curved axis of the city. The figure also shows the street spacing (left) along two roughly orthogonal profiles or transverses ( $a$ and $b$ ) in subarea/subpopulation 1-5 (modified from Mohajeri 2012).

of streets. When the data are shown on $\log -\log$ plots (Figure $6 \mathrm{~b}$ and $\mathrm{c}$ ), an abrupt break in slope occurs at about the same street length as in Dundee $(120 \pm 20 \mathrm{~m})$. The first population is composed of primarily local streets with lengths from $3 \mathrm{~m}$ to $120 \pm 20 \mathrm{~m}$, and the second of collector and minor arterial streets as long as $1192 \mathrm{~m}$.

The variation in spacing between streets in Khorramabad was analysed to see what effects, if 


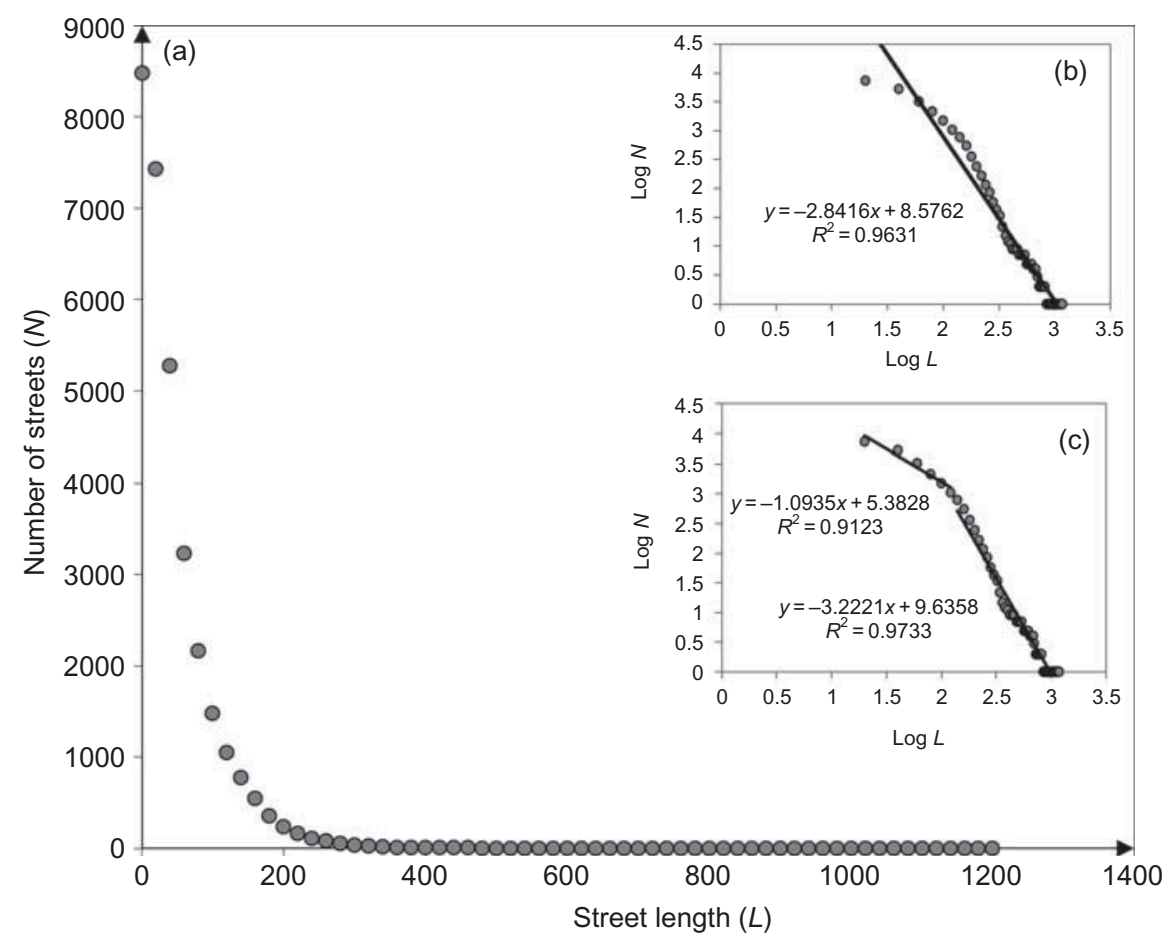

Figure 6. Power law frequency plot of all street segments (8481) measured in Khorramabad. (a) Ordinary cumulative length distribution. (b) Single-line $\log -\log$ fit. (c) Double-line $\log -\log$ fit. The different scaling exponents indicate different street populations.

any, its very clear landscape constraints have on street density and functionality. Street density is the reciprocal of spacing and is defined as the number of streets per unit length of a transverse roughly perpendicular to the mean trend of the streets. Spacing is defined as the shortest distance between the central lines (or middle parts) of adjacent streets. Variation in the street spacing or density indicates how the capacity for traffic transport may change within a city (Mohajeri 2012). For example, a narrow valley may form a bottleneck where the street spacing would be expected to decrease or the density to increase, to maintain uniform capacity for traffic flow along the city (Figure 5-right). In each subarea (Figure 5-left), the spacing along two roughly orthogonal lines or transverses was measured; one, marked by $a$, is parallel with the dominating (easterly) main trend of streets; the other, $b$, is parallel with the subordinate main trend. The two transverses are roughly perpendicular to the trends of the streets for which spacing are determined.

Several points emerge from the results in Table 1. First, the street spacing follows approximately normal distributions. The standard deviations vary but are much smaller for the easterly streets (i.e. those crossing lines $b$ ). Second, the spacing is also, on average, much less for the streets crossing lines $b$ than for those crossing lines $a$ as revealed in Table 1. The mean spacing in the subareas or subpopulations for the streets crossing lines $b$ varies from $27.82 \mathrm{~m}$ to $60.84 \mathrm{~m}$, with an average mean spacing value of about $50.71 \mathrm{~m}$. By contrast, the mean spacing for the streets crossing lines $a$ in the same subareas varies from $52.13 \mathrm{~m}$ to $123.26 \mathrm{~m}$, with an average mean spacing of about $106.15 \mathrm{~m}$. Thus, the mean spacing of the streets that cross lines $a$, and are thus parallel with the axis of the elongated, crescent city, is roughly twice that of the streets crossing the lines $b$. The easterly trending streets, that is, those crossing lines $b$, have a much higher density (much less spacing) than the streets crossing lines $a$. Third, the minimum spacing occurs in subarea/subpopulation 3: shown in bold (Table 1, Figure 5), namely at the narrowest part, or the bottleneck, of the city. The low average street spacing, or high street density, in this subarea is a further indication that the external landscape influences not only the overall shape of the city but also its internal street pattern.

\section{Critical testing of power law distributions}

Although the practice of attributing good straight line fits of $\log -\log$ distributions to the existence of an underlying power law process is widespread, it is entirely possible that other distributions may provide a better statistical fit and more closely represent the underlying generative process. In short, much of the analysis of power law relationships is based on visual analysis of the $\log -\log$ plot rather than any serious consideration of other possible relations that also show good visual fits but are very different from the 
Table 1. Number of street-spacing measurements, mean spacing, standard deviations and minimum and maximum spacing in subareas/subpopulations 1-5, Khorramabad, along transverses/profiles a and b in each subarea (located in Figure 7-right).

\begin{tabular}{|c|c|c|c|c|c|c|c|}
\hline \multicolumn{8}{|c|}{ Street-segments spacing } \\
\hline Profile & $\begin{array}{c}\text { Profile } \\
\text { orientation }\end{array}$ & $\begin{array}{l}\text { Street } \\
\text { trend }\end{array}$ & $\begin{array}{l}\text { Number of } \\
\text { spacing }\end{array}$ & $\begin{array}{c}\text { Mean } \\
\text { spacing }(\mathrm{m})\end{array}$ & $\begin{array}{l}\text { Standard } \\
\text { deviation }\end{array}$ & $\begin{array}{l}\text { Minimum } \\
\text { spacing }(\mathrm{m})\end{array}$ & $\begin{array}{l}\text { Maximum } \\
\text { spacing }(m)\end{array}$ \\
\hline$a-1$ & SW-NE & NW & 15 & 121.73 & 71.98 & 17 & 323 \\
\hline b-1 & SE-NW & $\mathrm{NE}$ & 49 & 53.92 & 21.43 & 22 & 137 \\
\hline$a-2$ & SW-NE & NW & 19 & 123.26 & 55.41 & 23 & 253 \\
\hline$b-2$ & SE-NW & $\mathrm{NE}$ & 45 & 51.73 & 11.3 & 27 & 89 \\
\hline a-3 & $\mathbf{W}-\mathbf{E}$ & $\mathbf{N}$ & 39 & 52.13 & 31.28 & 13 & 163 \\
\hline b-3 & $\mathbf{S}-\mathbf{N}$ & $\mathbf{E}$ & 72 & 27.82 & 11.49 & 8 & 75 \\
\hline$a-4$ & SE-NW & $\mathrm{NE}$ & 12 & 122.83 & 66.67 & 34 & 205 \\
\hline$b-4$ & SW-NE & WNW & 34 & 59.24 & 15.48 & 33 & 99 \\
\hline$a-5$ & SE-NW & $\mathrm{NE}$ & 19 & 110.79 & 54.89 & 38 & 239 \\
\hline$b-5$ & SW-NE & NW & 32 & 60.84 & 28.98 & 26 & 166 \\
\hline
\end{tabular}

simplest power law case. Therefore, following the methods advocated by Newman (2005) and Clauset, Shalizi, and Newman (2009), maximum likelihood estimators (MLE) with goodness of fit tests based on Kolmogorov-Smirnov statistic and likelihood ratios were used to evaluate the power law behaviour apparent from visual examination of the street network data. This method permits estimation of the scaling exponent $(\alpha)$, and also the lowermost or minimum value $\left(x_{\min }\right)$ down to which the distribution follows a power law. Following Clauset, Shalizi, and Newman (2009), a quantity $\times$ obeys a continuous power law distribution if it is drawn from a probability density function or PDF such that:

$$
P(x)=C x^{-a}
$$

where $C$ is a normalised constant based on the minimum value or lower-bound of the power law $\left(x_{\min }\right)$ and $\alpha$ is the scaling exponent. Generally, a power law fit to empirical data does not apply for all $x \in x \geq 0$. There must be some lower bound or minimum value for the power law fit. Often, a power law fit applies only to data larger than $x_{\min }$, i.e. to the tail of the distribution. It follows that a definition of power law distribution, using normalisation, is:

$$
P(x)=\frac{\alpha-1}{x_{\min }}\left(\frac{x}{x_{\min }}\right)^{-\alpha}
$$

Using Equation (7), we can estimate $\alpha$ thus:

$$
\alpha=1+n\left[\sum_{i=1}^{n} \ln \frac{x_{i}}{x_{\min }}\right]^{-1}
$$

where $x_{i}, i=1 \ldots n$ are the observed values of $\times$ such that $x_{i} \geq x_{\min }, \alpha$ is the slope of the line in the power law domain, $n$ is now the number of data bins used in the calculations (excluding those with values below $x_{\min }$ ), and $x_{\min }$ is the lower bound for the power law fit to apply. It may be helpful to explore the complementary cumulative distribution function (CDF) of a power law distribution function. The shape or form of the CDF normally shows less fluctuation than that of the PDF, in particular in the tail of the distribution (Newman 2005). The cumulative distribution function $P(x)$ in relation to the probability distribution $P r$ is defined as $P(x)=\operatorname{Pr}(X \geq x)$. For the continuous case, the formula is (Clauset, Shalizi, and Newman 2009):

$$
P(x)=\int_{x}^{\infty} P\left(x^{\prime}\right) d x^{\prime}=\left(\frac{x}{x_{\min }}\right)^{-\alpha+1}
$$

where $x_{i}, i=1 \ldots n$ are the observed values of $\times$ such that $x_{i} \geq x_{\min }$. In the present analysis, $x_{\min }$ is chosen so as to make the cumulative distributions of the measured data and the best-fit power law as similar for $x_{i} \geq x_{\min }$. There are a variety of methods for quantifying the distance between two distribution functions, but for nonnormally distributed data, the most common method is the Kolmogorov-Smirnov or KS statistic, which is the maximum distance between a distribution function (CDF) of the data and the fitted model. This is defined as:

$$
\Delta=\max _{x \geq x \min }|S(x)-P(x)|
$$

Here, $S(x)$ is the CDF of the data for the observations with values larger than or equal to $x_{\min }$, and $P(x)$ is the CDF for the power law that best fits the data in the region $x \in \geq x_{\min }$. Our estimate of $x_{\min }$ is then the value of $x_{\min }$ that minimises $\Delta$ in Equation (10).

When $x_{\min }$ and $\alpha$ have been calculated, we can find the goodness-of-fit between the data and the power law. A goodness-of-fit test generates a $P$-value that quantifies the plausibility of the hypothesis that the data fit a power law. It should be noted that a large $P$-value does not necessarily mean that a power law is the best model for the data. 
First, there may be other models or distributions that match (fit) the data equally well or better over the observed range of $x$. Second, for a small number of data, it is very difficult to rule out a power law model; even if the calculated $P$-value is large, the power law fit may be spurious.

To explore these points further, power law models for the street network data are compared with alternative models using a likelihood ratio test. For each alternative model (fit), if the calculated likelihood ratio is significantly different from zero, then its sign indicates whether the alternative is favoured over the power law model. To do so, we calculate the logarithm of the likelihood ratio $(R)$, which has a positive and negative sign depending on which distribution is better, or zero if the model fits are equally good. More specifically, positive values of the log-likelihood ratio indicate that the power law model is favoured over the alternative. However, the sign of the $R$ alone is not sufficient to determine which model provides the better fit because, like other quantities, the ratio is subject to statistical fluctuations. To make an objective judgement as to whether the observed value of $R$ is sufficiently far from zero, we need to know the size of the expected statistical fluctuations, that is, the standard deviation $\sigma$ of $R$. To estimate $\sigma$, we use a method used by Clauset, Shalizi, and Newman (2009) that gives a $P$-value that tells us whether the observed sign of $R$ is statistically significant.

Using the maximum likelihood method for testing the appropriateness of power law models can, however, be problematic (Newman 2005; Clauset, Shalizi, and Newman 2009). For example, it is very difficult to decide between log-normal and the power law models because, for realistic ranges of $x$, the two models are very similar. It is therefore unlikely that any test would be able to discriminate between these models unless the data set is very large. Also, in many cases, the results from comparing power laws with other distributions based on calculations of $P$-value and likelihood ratio tests does not help us to decide which model fits better with the data.

When a decision cannot be made using quantitative approach, the final decision as to which model best fits the data may have to be based on our intuition. The value of such a judgement about the best-fitting model for a data distribution can be greatly improved by considering the likely physical basis or theoretical factors that generate, or contribute to the generation of, the data. More specifically, we should consider physical, that is, the non-statistical arguments that might favour one model fit over the alternative models. Thus, in many cases the decision as to whether to use a power law or an alternative model does not only depend on how well the models fit the data but also on the theoretical framework and the scientific aims of the study (Clauset, Shalizi, and Newman 2009; Huges and Hase 2010; Berendsen 2011).

Based on these considerations, maximum likelihood estimators were calculated for the power law fits to the realdata distributions. The goodness-of-fit was also calculated
Table 2. Tests of power law behaviour in the data sets.

\begin{tabular}{llcc}
\hline \multicolumn{3}{c}{ Tests of power law behaviour in street data sets } \\
\hline & & & Khorramabad, \\
& City & Dundee, 2007 & 2006 \\
\hline & $n$ & 9616 & 8481 \\
& $\alpha$ & $3.27 \pm 0.09$ & $3.81 \pm 0.42$ \\
& $n_{\text {tail }}$ & $961 \pm 241$ & $2751 \pm 1785$ \\
& $x_{\text {min }}$ & $165 \pm 26$ & $116 \pm 33$ \\
Power law & $P$ & 0.34 & 0.00 \\
Log normal & LR & -0.402 & -32.481 \\
& $P$ & 0.29 & 0.00 \\
Exponential & LR & 90.124 & -21.876 \\
& $P$ & 0.99 & 0.07 \\
Stretched & LR & -0.359 & -27.479 \\
exponential & & & 0.00 \\
& $P$ & 0.36 & 0 \\
\hline
\end{tabular}

Notes: Number of street segments for each city $(n)$, scaling exponent based on MLE $(\alpha)$ and the standard error of $\alpha$, the number of observation in the power law region (range) $\left(n_{\text {tail }}\right)$ and standard error of $n_{\text {tail }}$, lower bound of power law $\left(x_{\min }\right)$ at which the power law no longer applies and standard error of $x_{\min }$, power law fits and the corresponding $P$-values, a $P$-value for the fit to the power law model and likelihood ratios for the alternative models (fits). Positive values of the log-likelihood ratios indicate that the power law model is favoured over the alternative models if the $P$-value $<0.1$. However, if the $P$-value is larger than 0.1 , the sign is not reliable indicator of which model is the better fit to the data.

to estimate the lower cut-off $\left(x_{\min }\right)$ for the scaling region and the KS statistic (which computes a $P$-value for the estimated power law fit to the data). The uncertainty/error in the estimated parameters for the power law fit was also evaluated. However, to compute the log-likelihood ratios for two competing models (fits), freely available $R$ routines were used (http://tuvalu.santafe.edu/ aaronc/powerlaws/). The results are summarised in Table 2 . The $P$-values for the power laws indicate that the Dundee data set fits very well with a power law. However, the likelihood-ratio tests have $P$-values so large $(0.29,0.99,036)$ that they cannot be used to decide which of the various alternative models best fits data. In contrast, the data set of Khorramabad has $P$-value so small (effectively 0.0 ) that the power law model can be ruled out. In the likelihood-ratio test for Khorramabad, the $P$-value is small enough for the signs to be reliable; the results show that any of the other models are plausible.

Even if the alternative distributions (log-normal, exponential and stretched exponentials) may statistically fit some of the street network data sets better than a power law, power law fits may still be useful. For example, as shown in the present analysis, they provide a convenient basis for distinguishing between street subpopulations that have different functions. This kind of analysis is developed further with reference to the entropy concepts in the following section.

\section{Geometric evolutions of cities and entropy analysis}

We will now explore how the street patterns, as regards their lengths, can be interpreted with reference to concepts 
drawn from statistical mechanics/information theory, primarily based on entropy measures The focus is on the evolution of Dundee in the time periods from the seventeenth century to the year 2007 and the evolution of Khorramabad in the time periods from the 1955 to 2006.

Lengths of street segments were analysed for each time periods (Figures 7a and 8a). Plots of the cumulative distributions of street lengths (Figures $7 \mathrm{~b}$ and $8 \mathrm{~b}$ ) for different time periods provide different curves on the log-log plots (Figures 7c and 8c). In particular, in Dundee there are noticeable changes in the approximate straight-line slopes at about the same street lengths as in Khorramabad, that is, at $120 \pm 20 \mathrm{~m}$ (Tables 3 and 4). This indicates different street populations (using the same 'regression-line' fits as in Figures 4 and 6 but not shown in Figures 7c and $8 \mathrm{c})$. All the street populations for Dundee are shown in Table 3, and for Khorramabad is shown in Table 4, where 'breaks' in slope, marking the change from one population to another, occur at lengths from $100 \mathrm{~m}$ to $140 \mathrm{~m}$. Thus, the short-street populations range in length from $3 \mathrm{~m}$ to (a)

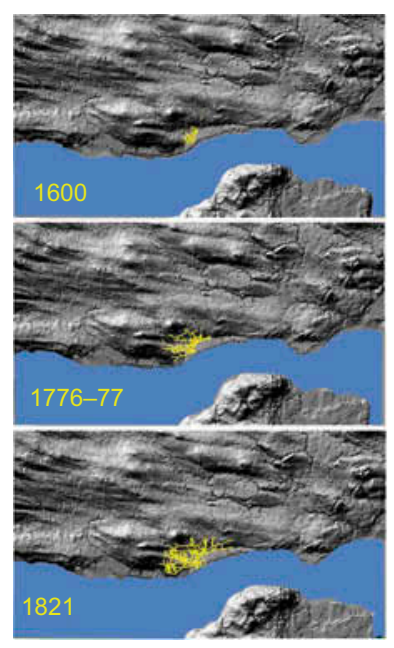

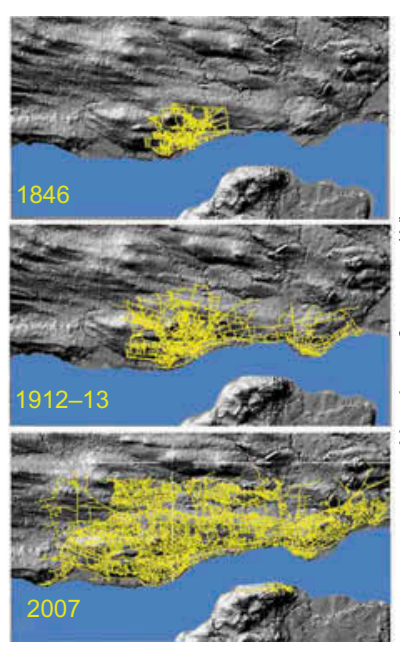

(b)

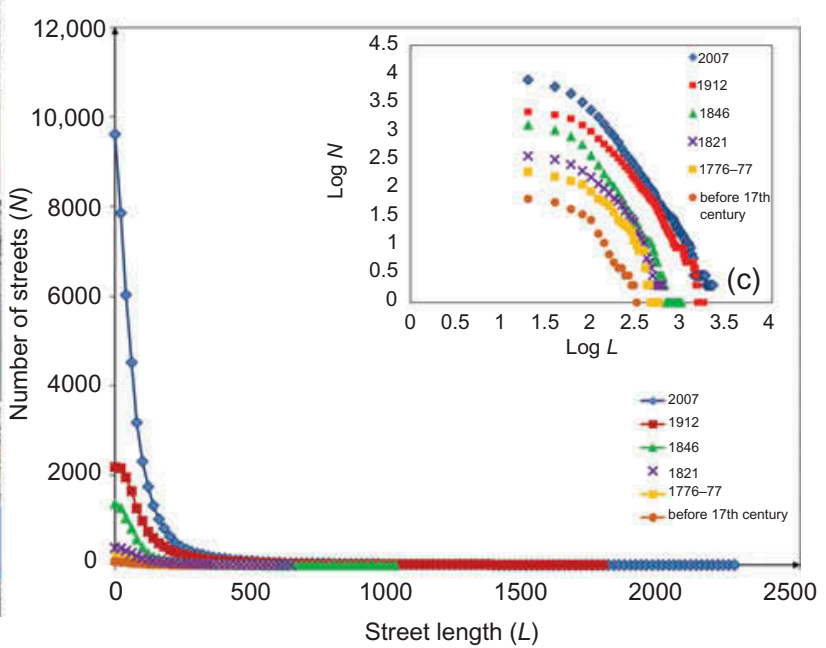

Figure 7. DEMs (Digital Elevation Models) showing the situation of Dundee (a) in the six sampled time periods from before seventeenth century to 2007, (b) cumulative length distributions of streets in Dundee during time from before seventeenth century to the year 2007 and (c) log-transformed plots of street number versus street length.

(a)

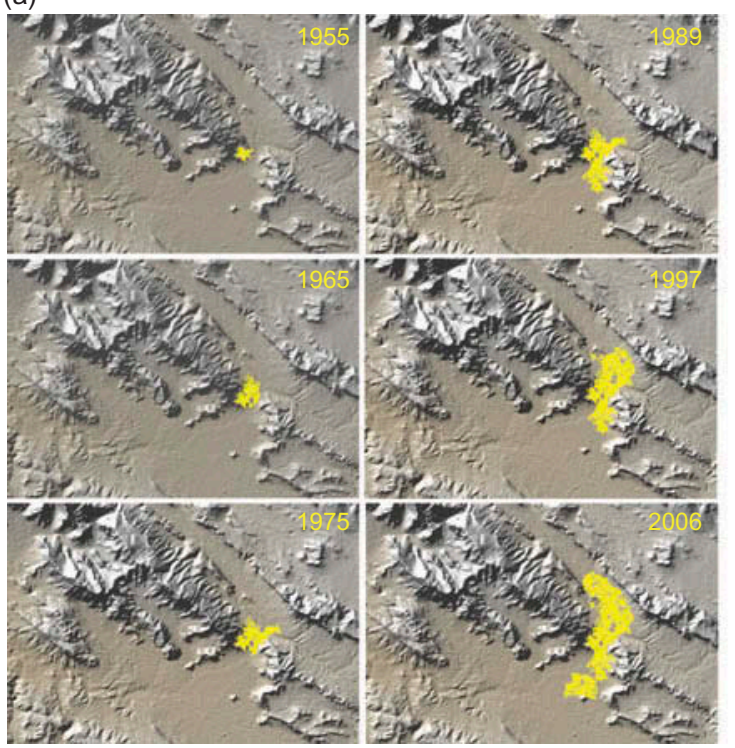

(b)

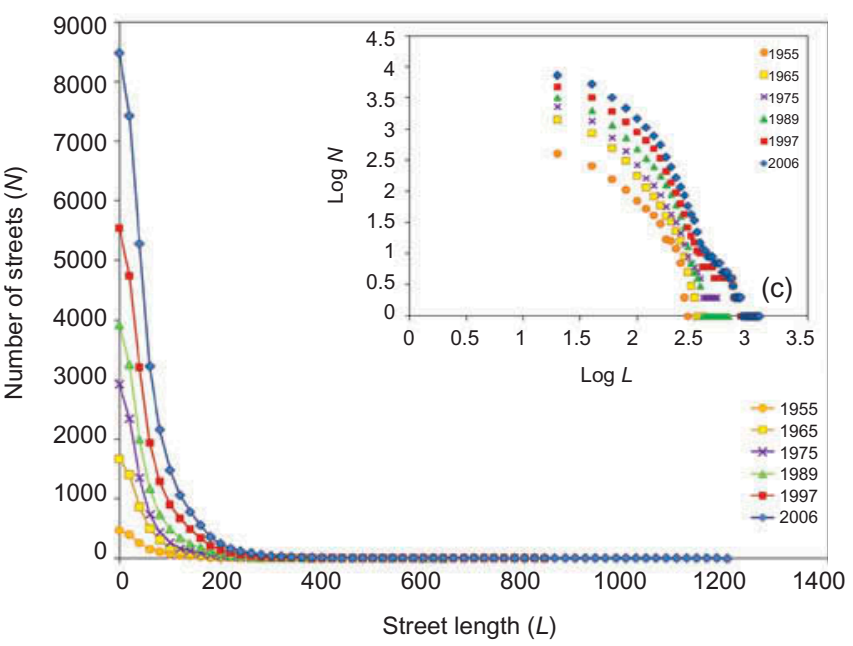

Figure 8. (a) DEMs (Digital Elevation Models) showing the geometry of Khorramabad (a) at the six sampled time periods from 1955 to 2006, (b) cumulative length distributions of streets in Khorramabad during time from 1955 to the year 2006 and (c) log-transformed plots of street number versus street length. 
Table 3. Number or frequency, length range, average length, scaling exponent $(D)$, coefficient of determination $\left(R^{2}\right)$ and entropy $(S)$ of the populations and subpopulations during the evolution of the city of Dundee.

\begin{tabular}{|c|c|c|c|c|c|c|}
\hline \multicolumn{7}{|c|}{ Street segments (Dundee) } \\
\hline Year & Frequency & $\begin{array}{l}\text { Length range } \\
\qquad(m)\end{array}$ & $\begin{array}{l}\text { Average } \\
\text { length }\end{array}$ & $\begin{array}{l}\text { Scaling exponent } \\
\text { (D) }\end{array}$ & $R^{2}$ & $\begin{array}{c}\text { Entropy } \\
(S)\end{array}$ \\
\hline 1600 & 70 & $12-336$ & 92 & 1.594 & 0.888 & 2.245 \\
\hline $\mathbf{A}$ & - & 12-100 & 52 & 0.527 & 0.921 & 1.741 \\
\hline B & - & $100-336$ & 153 & 2.428 & 0.956 & 2.114 \\
\hline $1776-17$ & 209 & $9-569$ & 113 & 1.898 & 0.814 & 2.60 \\
\hline $\mathbf{A}$ & - & 9-100 & 52 & 0.451 & 0.931 & 1.751 \\
\hline B & - & $100-569$ & 196 & 3.157 & 0.913 & 2.548 \\
\hline 1821 & 376 & $12-625$ & 117 & 1.908 & 0.860 & 2.613 \\
\hline $\mathbf{A}$ & - & $12-120$ & 65 & 0.603 & 0.875 & 1.875 \\
\hline B & - & $120-625$ & 228 & 3.110 & 0.957 & 2.608 \\
\hline 1846 & 1360 & $8-1008$ & 88 & 2.43 & 0.939 & 2.337 \\
\hline $\mathbf{A}$ & - & 8-100 & 55 & 0.702 & 0.868 & 1.710 \\
\hline B & - & $100-1008$ & 171 & 2.995 & 0.971 & 2.639 \\
\hline $1912-1913$ & 2184 & $11-1770$ & 130 & 2.166 & 0.928 & 2.862 \\
\hline A & - & $11-120$ & 70 & 0.575 & 0.841 & 1.883 \\
\hline B & - & $120-1770$ & 248 & 2.641 & 0.963 & 3.224 \\
\hline 2007 & 9616 & $3-2248$ & 80 & 2.262 & 0.968 & 2.539 \\
\hline A & - & $3-140$ & 53 & 0.917 & 0.922 & 1.889 \\
\hline B & - & $140-2248$ & 254 & 2.559 & 0.984 & 3.268 \\
\hline
\end{tabular}

Note: A and B (in bold) refer to subpopulations of short and long streets, respectively.

Table 4. Number or frequency, length range, average length, scaling exponent $(D)$, coefficient of determination $\left(R^{2}\right)$ and entropy $(S)$ of the populations and subpopulations during the evolution of the city of Khorramabad.

\begin{tabular}{|c|c|c|c|c|c|c|}
\hline \multicolumn{7}{|c|}{ Street segments (Khorramabad) } \\
\hline Year & Frequency & $\begin{array}{l}\text { Length range } \\
\qquad(m)\end{array}$ & $\begin{array}{l}\text { Average } \\
\text { length }\end{array}$ & $\begin{array}{l}\text { Scaling exponent } \\
\text { (D) }\end{array}$ & $R^{2}$ & $\begin{array}{l}\text { Entropy } \\
(S)\end{array}$ \\
\hline 1955 & 468 & $4-269$ & 60 & 1.818 & 0.861 & 1.967 \\
\hline $\mathbf{A}$ & - & $4-100$ & 43 & 1.065 & 0.960 & 1.601 \\
\hline B & - & $100-269$ & 160 & 3.648 & 0.865 & 1.799 \\
\hline 1965 & 1671 & $3-375$ & 54 & 2.634 & 0.875 & 1.846 \\
\hline $\mathbf{A}$ & - & $3-140$ & 47 & 1.490 & 0.942 & 1.684 \\
\hline B & - & $100-375$ & 197 & 5.443 & 0.958 & 1.859 \\
\hline 1975 & 2927 & $3-598$ & 50 & 2.750 & 0.951 & 1.809 \\
\hline $\mathbf{A}$ & - & $3-100$ & 39 & 1.333 & 0.955 & 1.517 \\
\hline B & - & $100-598$ & 154 & 3.543 & 0.981 & 2.163 \\
\hline 1989 & 3932 & $4-638$ & 55 & 3.121 & 0.904 & 1.911 \\
\hline A & - & $4-140$ & 46 & 1.342 & 0.960 & 1.719 \\
\hline B & - & $140-638$ & 195 & 4.812 & 0.928 & 1.952 \\
\hline 1997 & 5535 & $3-882$ & 62 & 2.656 & 0.946 & 2.020 \\
\hline A & - & $3-120$ & 47 & 1.109 & 0.956 & 1.711 \\
\hline B & - & $120-882$ & 176 & 3.078 & 0.938 & 2.157 \\
\hline 2006 & 8481 & $3-1192$ & 65 & 2.841 & 0.963 & 2.053 \\
\hline A & - & $3-120$ & 49 & 1.093 & 0.942 & 1.726 \\
\hline B & - & $120-1192$ & 179 & 3.222 & 0.973 & 2.227 \\
\hline
\end{tabular}

Note: A and B (in bold) refer to subpopulations of short and long streets, respectively.

$120 \pm 20 \mathrm{~m}$ for both cities, whereas the long-street segments range from $120 \pm 20 \mathrm{~m}$ to $2248 \mathrm{~m}$ for Dundee and to $1192 \mathrm{~m}$ for Khorramabad. Using these results, the scaling exponents and the length ranges of the street populations from Dundee and Khorramabad can be compared with their entropies.
The length distribution of streets is a measure of the associated entropy. This follows because entropy in the probabilistic sense is an indication of the spread of any kind of frequency distribution (Dill and Bromberg 2003; Volkenstein 2009). Equation (3) can be used to calculate the entropies associated with the various street populations, 

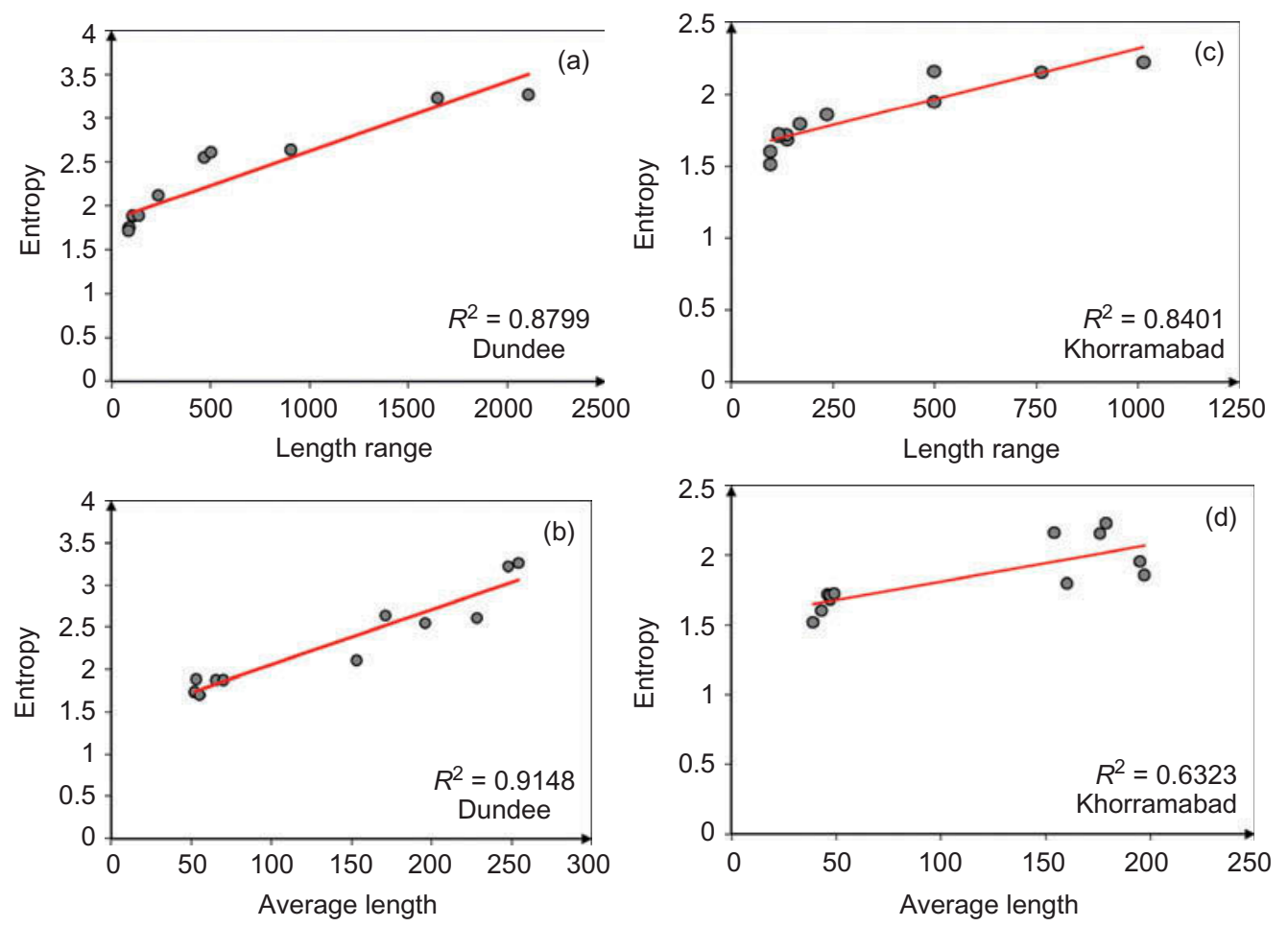

Figure 9. Entropy versus the length range and average length of streets in Dundee and Khorramabad. (a and b) Correlations for the short (populations marked by ' $A$ ' in Table 3 ) and long (tail) populations (those marked by ' $A$ ' in Table 3). (c and d) Correlations for the short populations (marked by 'A' in Table 4) and long (tail) populations (marked by 'B' in Table 4). Only the subpopulations shown in Tables 3 and 4 are plotted since the whole populations do not fit with straight-line plots.

as well as the scaling exponents of the power laws, $D$, as in Equation (1).

Entropies, scaling exponents, length ranges and average lengths of street populations in Dundee and Khorramabad are compared in Tables 3 and 4 . Considering first the relations among the populations (A, B) from Tables 3 and 4, the results are plotted in Figure 9. There is clearly a strong positive correlation between the entropies and (a) the length ranges and (b) the average lengths of the streets in these populations as a function of time, that is, during the evolution of the city. It is clear that short streets are of less importance in these relations than the long streets because the maximum (and average) lengths of the short streets do not change much with the expansion of the city. Thus, the focus is on the long streets (steep-slope or that tailpart) populations, since these are likely to change with the growth of the street network. Clearly, all the three parameters (entropy, length range and average length) increase as the street network expands during the growth of these cities. This implies that as the city grows, the tail populations increase their average and maximum lengths and thus become more dispersed or spread, thereby increasing their entropies.

The maximum likelihood method is again used to test whether the data for each of the city-evolution periods are consistent with power laws, using Dundee as an example. Results (see the $P$-values in Table 5) indicate that most of the Dundee-evolution data sets are indeed consistent with power law models, the exception being the data for 1776-17. However, $P$-values for the alternative models are so large that we cannot decide which, if any, of the alternative models are statistically better. This also applies to the other time periods, with the exceptions of 1821 and 1912 , where the $P$-values are small enough for the signs to be reliable such that log-normal, exponential and stretched exponential models also provide plausible fits as shown in Table 5.

\section{Discussion and conclusion}

Before the industrial revolution (which began in Scotland in the early nineteenth century), the centre of Dundee has preserved most of its character from medieval times The street patterns particularly in the southern and eastern parts still follow an irregular layout, similar to the old medieval town. Thus, the city grew until that time in a sort of natural way through 'bottom-up', individualistic rather than 'top-down' institutionalised collective processes. However, many of the medieval local streets were demolished in the late nineteenth century for extension of the Victorian streetscape (Ferguson 2005; Watson 2006). By contrast, the city of Khorramabad is an example of a rapidly expanding city, with most of its streets being planned by the central government and in a 'top-down' manner (Ministry of 
Table 5. Tests of power law behaviour of Dundee-evolution data sets.

\begin{tabular}{|c|c|c|c|c|c|c|c|}
\hline \multicolumn{8}{|c|}{ Tests of power law behaviour in street data sets } \\
\hline \multicolumn{2}{|l|}{ Dundee evolution } & Before 1700 & $1776-17$ & 1821 & 1846 & 1912 & 2007 \\
\hline \multicolumn{2}{|l|}{$\mathrm{n}$} & 70 & 209 & 376 & 1360 & 2184 & 9616 \\
\hline \multicolumn{2}{|l|}{$\alpha$} & $3.46 \pm 0.39$ & $2.79 \pm 0.42$ & $2.71 \pm 0.43$ & $3.33 \pm 0.14$ & $2.93 \pm 0.27$ & $3.27 \pm 0.09$ \\
\hline \multicolumn{2}{|l|}{$n_{\text {tail }}$} & $32 \pm 11$ & $93 \pm 30$ & $185 \pm 71$ & $440 \pm 110$ & $615 \pm 358$ & $961 \pm 241$ \\
\hline \multicolumn{2}{|l|}{$x_{\min }$} & $101 \pm 23$ & $108 \pm 37$ & $103 \pm 50$ & $100 \pm 15$ & $164 \pm 57$ & $165 \pm 26$ \\
\hline \multirow{3}{*}{$\begin{array}{l}\text { Power law } \\
\text { Log normal }\end{array}$} & $P$ & 0.99 & 0.00 & 0.40 & 0.32 & 0.21 & 0.34 \\
\hline & LR & 0.000 & -3.388 & -8.461 & -1.198 & -4.181 & -0.402 \\
\hline & $P$ & 0.57 & 0.22 & 0.00 & 0.17 & 0.04 & 0.29 \\
\hline \multirow[t]{2}{*}{ Exponential } & LR & 3.048 & -5.044 & -9.436 & 19.214 & 24.124 & 90.124 \\
\hline & $P$ & 0.89 & 0.11 & 0.00 & 0.98 & 0.96 & 0.99 \\
\hline \multirow[t]{2}{*}{ Stretched exponential } & LR & 0.048 & -5.080 & -9.520 & -1.279 & -4.169 & -0.359 \\
\hline & $P$ & 0.63 & 0.11 & 0.00 & 0.17 & 0.04 & 0.36 \\
\hline
\end{tabular}

Notes: Number of street segments for each time period $(n)$, scaling exponent based on MLE $(\alpha)$ and standard error of $\alpha$, the number of observation in the power law region (range) ( $\left.n_{\text {tail }}\right)$ and standard error of $n_{\text {tail }}$, lower bound of power law $\left(x_{\min }\right)$ at which the power law no longer applies and standard error of $x_{\min }$, power law models (fits) and the corresponding $P$-values, a $P$-value for the fit to the power law model and likelihood ratios for the alternative models.

Housing and Urban Development 2005; Mohajeri 2012). As the growth of cities is a complex process, affected by many parameters, it is clear that Dundee and Khorramabad have evolved through different processes. However, even if the growth processes have been different, with initial street patterns quite different, the contemporary street patterns show many similarities (Levinson and Huang 2012).

The results presented here show, first, that landscape constraints can have large effects on the general city boundary shape and, second, that boundary shape, in turn, affects the street patterns. In the case of Dundee, street lengths are to a large degree controlled by their orientation in relation to the estuary shoreline that bounds the city to the south (Figures 3 and 5). Coast-parallel streets are longer and most streets are either perpendicular or parallel to the shore. In Khorramabad, the mountain slopes continue to constrain city shape as well as trend and length evolution of streets (Figure 5). These constraints are reflected not only in the trends of the streets but also in the minimum street spacing being in the narrowest part of the city (Table 1).
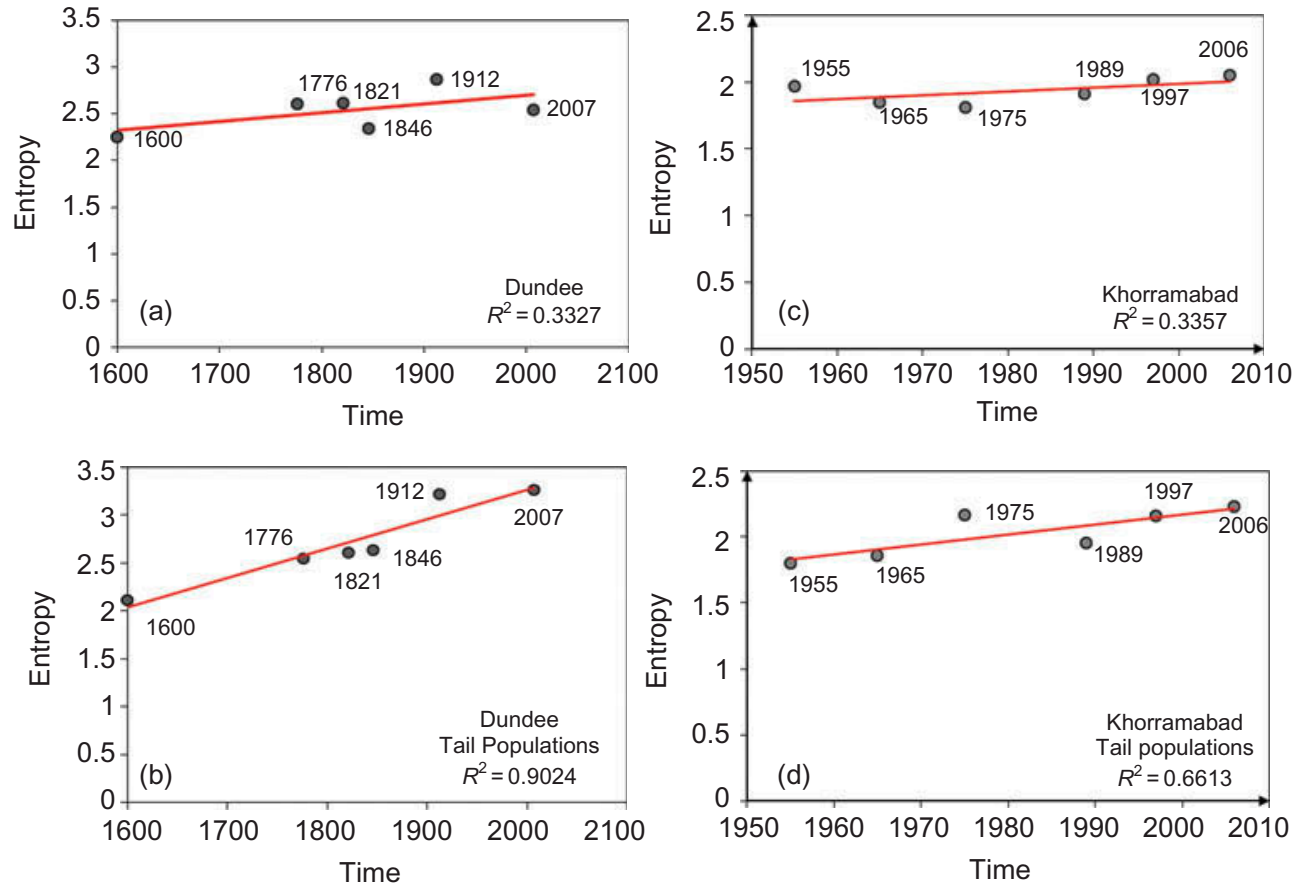

Figure 10. The entropy of the whole street-length populations $(a, b)$ as a function of time has remained essentially constant during the sampled time periods. By contrast, the entropy of the long (tail) length populations has increased during the sampled time periods (c, d). 
Most of the street populations show power law length distributions with the obvious point there are many more short streets than long ones. The power law size distributions of street networks, or city size, indicate that a city system self-organises itself as a hierarchical structure at different scales, from the smallest to the largest (Zipf 1949; Simon 1955; Salingaros 2005; Batty 2006, 2008; Pumain 2006; Jiang 2007; Levinson and Huang 2012). On $\log -\log$ plots, the street populations of both cities show breaks in the straight-line slopes (yielding different scaling exponents D) at roughly the same street lengths, namely at $120 \pm 20 \mathrm{~m}$. The shorter streets are mainly local streets whereas the longer ones are collectors and arterials (Figures 7 and 8).

A long power law tail (subpopulations B in Tables 3 and 4) normally implies a more dispersed (spread) distribution and thus a high entropy (in comparison with subpopulations A). To test this implication, the entropies calculated for the Dundee and Khorramabad street subpopulations were plotted against their length ranges and average lengths, considering the sampled time periods (Figure 9). The results show a linear correlation between the calculated entropies and the length ranges and average lengths of these subpopulations, implying that the entropy of a street network increases over time with increase in its maximum and average street lengths. The results in Figure 10 suggest that the entropy of neither city changes significantly over the six sampled time periods. However, the entropies of the long (tail) populations have a high correlation and clearly increase with time.

In conclusion, the two case studies, the cities of Dundee and Khorramabad, clearly show how their shapes and associated street patterns are constrained by the surrounding landscape. The entropy analysis method presented here is used to quantify the variation in street trends and lengths in relation to landscape constraints within cities and between cities as well as city evolutions. We believe that this method, as demonstrated by the results presented here, offers great possibilities for quantifying not only other properties of street patterns such as width, density and connectivity, but also for other linear spatial structures in cities, obviously other kinds of networks that can be coupled to street patterns but also clusters of related locations that are linked through more abstract relations.

\section{References}

Barredo, J. I., M. Kasanko, N. McCormick, and C. Lavalle. 2002. "Modelling Dynamic Spatial Processes: Simulation of Urban Future Scenarios Through Cellular Automata." Landscape and Urban Planning 64: 145-160.

Barthelemy, M., and A. Flammini. 2008. "Modeling Urban Street Patterns." Physical Review Letters 100: 138702.
Batty, M. 1971. "Modelling Cities as Dynamic Systems." Nature 231: 425-428.

Batty, M. 2006. "Hierarchy in Cities and City Systems." In Hierarchy in the Natural and Social Sciences, edited by D. Pumain, 143-168. Dordrecht: Springer.

Batty, M. 2008. "Cities as Complex Systems: Scaling, Interactions, Networks, Dynamics and Urban Morphologies." Working Papers Series No. 131. Centre for Advanced Spatial Analysis, University College London.

Batty, M. 2010. "Space, Scale, and Scaling in Entropy Maximizing." Geographical Analysis 42: 395-421.

Benguigui, L., E. Blumenfeld-Lieberthal, and D. Czamanski. 2006. "The Dynamics of the Tel Aviv Morphology." Environment and Planning B 33: 269-284.

Ben-Naim, A. 2008. A Farewell to Entropy: Statistical Thermodynamics Based on Information Theory. Singapore: World Scientific Publishing.

Berechman, J., and K. A. Small. 1988. "Modeling Land Use and Transportation: An Interpretive Review for Growth Areas." Environment and Planning A 20: 1285-1309.

Berendsen, H. J. C. 2011. Data and Error Analysis. Cambridge: Cambridge University Press.

Berling, S. H., and J. Wu. 2004. "Modeling Urban Landscape Dynamics: A Review." Ecological Research 19: 119-129.

Blundell, S. J., and K. M. Blundell. 2006. Concepts in Thermal Physics. Oxford: Oxford University Press.

Cardillo, A., S. Scellato, V. Latora, and S. Porta. 2006. "Structural Properties of Planar Graphs of Urban Street Patterns." Physical Review E 73: 1-8.

Chen, Y. G. 2012. "The Rank-Size Scaling Law and EntropyMaximizing Principle." Physica A 391: 767-778.

Clauset, A., C. R. Shalizi, and M. E. J. Newman. 2009. "Power Law Distributions in Empirical Data." SIAM Review 51: 661-703.

Desurvire, E. 2009. Classical and Quantum Information Theory. Cambridge: Cambridge University Press.

Dill, K. A., and S. Bromberg. 2003. Molecular Driving Forces: Statistical Thermodynamics in Chemistry and Biology. London: Taylor and Francis.

Drissi, N., T. Chonavel, and J. M. Boucher. 2008. "Generalized Cumulative Residual Entropy for Distributions With Unrestricted Supports." Research Letters in Signal Processing 790607: 5. doi:10.1155/2008/790607.

Ferguson, G. N. 2005. Dundee: A History and Celebration of the City. Dinton: Francis Firth.

General Register Office for Scotland. 2009. Census Data. Edinburgh: General Register Office for Scotland.

Harris, B. 1985. "Urban Simulation Models in Regional Science." Journal of Regional Science 25: 545-567.

Headicar, P. 2009. Transport Policy and Planning in Great Britain. London: Taylor and Francis.

Hillier, B. 1999. "The Hidden Geometry of Deformed Grids." Environment and Planning B 26: 169-191.

Huges, I. G., and T. P. A. Hase. 2010. Measurements and Their Uncertainties. Oxford: Oxford University Press.

Iranian Statistical Centre. 2007. Census Data. Tehran: Iranian Statistical Centre.

Jaynes, E. T. 1957. "Information Theory and Statistical Mechanics." Physical Review 106: 620-630.

Jiang, B. 2007. "Topological Pattern of Urban Street Networks: Universality and Peculiarity." Physica A 384: 647-655.

Jiang, B. 2009. "Street Hierarchies: A Minority of Streets Account for a Majority of Traffic Flow." International Journal of Geographic Information Science 23: 1033-1048.

Kondepudi, D., and I. Prigogine. 1998. Modern Thermodynamics. New York: Wiley. 
Lammer, S., B. Gehlsen, and D. Helbing. 2006. "Scaling Laws in the Spatial Structure of Urban Road Networks." Physica A 36: 89-95.

Levinson, D., and A. Huang. 2012. "A Positive Theory of Network Connectivity." Environment and Planning B 39: 308-325.

Makse, H. A., S. Havlin, and H. E. Stanley. 1995. "Modeling Urban Growth Patterns." Nature 377: 608-612.

Marshall, S. 2005. Streets and Patterns. London: Spon Press.

Masucci, A. P., D. Smith, A. Crooks, and M. Batty. 2009. "Random Planar Graphs and the London Street Network." European Physical Journal B 71: 259.

Ministry of Housing and Urban Development. 2005. Master Plan of Khorramabad. Tehran: Centre Information Iran.

Mohajeri, N. 2012. "Effects of Landscape Constraints on Street Patterns in Cities: Examples from Khorramabad, Iran." Applied Geography 34: 10-20.

Mohajeri, N., and A. Gudmundsson. 2012. "Entropies and Scaling Exponents of Street and Fracture Networks." Entropy 14: 800-833.

National Cartographic Centre of Iran. 2005. Digital Data of Khorramabad. Tehran: Tehran Cartographic Centre.

Navarro, J., Y. D. Aguila, and M. Asadi. 2010. "Some New Results on the Cumulative Residual Entropy." Journal of Statistical Planning and Inference 140: 310-322.

Nelson, P. 2006. Biological Physics: Energy, Information, Life. New York: W.H. Freeman.

Newman, M. E. J. 2005. "Power Laws, Pareto Distributions and Zipf's law." Contemporary Physics 46: 323-351.

Peitgen, H. O., H. Jurgens, and D. Saupe. 2004. Chaos and Fractals. New York: Springer-Verlag.

Pont, M. B., and P. Haupt. 2010. SpaceMatrix: Space, Density and Urban Form. Rotterdam: NAi.

Pumain, D, ed. 2006. Hierarchy in Natural and Social Sciences. Dordrecht: Springer.

Rao, M., Y. Chen, B. C. Vemuri, and F. Wang. 2004. "Cumulative Residual Entropy: A New Measure of
Information." IEEE Transactions on Information Theory 50: $1220-1228$

Salingaros, N. A. 2005. Principles of Urban Structure. Amsterdam: Techne Press.

Scellato, S., A. Cardillo, V. Latora, and S. Porta. 2006. "The Backbone of a City." European Physical Journal B 5: 221-225.

Schroeder, M. 1991. Fractals, Chaos, Power Laws. New York: W.H. Freeman.

Smith, M. J., M. F. Goodchild, and P. Longley. 2009. Geospatial Analysis: A Comprehensive Guide. London: Troubador.

Simon, H. A. 1955. "On a Class of Skew Distribution Functions." Biometrica 42: 425-440.

Stamps, A. E. 2004. "Entropy and Visual Diversity in the Environment." Journal of Architectural and Planning Research 21: 239-256.

Swan, A. R. H., and M. Sandilands. 1995. Introduction to Geological Data Analysis. Oxford: Blackwell.

Volkenstein, M. V. 2009. Entropy and Information. Berlin, DE: Birkhauser.

Wang, F., B. C. Vemuri, M. Rao, and Y. Chen. 2003. "Cumulative Residual Entropy, a New Measure of Information and Its Application to Image Alignment." Proceedings of the 9th International Conference on Computer Vision, 2 Volume Set 0-7695-1950-4/03, Nice, France, October 14-17.

Watson, N. 2006. Dundee: A Short History. London: Black and White.

Wegener, M. 1994. "Operational Urban Models State of the Art." Journal of the American Planning Association 60: 17-29.

Xie, F., and D. Levinson. 2007. "Measuring the Structure of Road Networks." Geographical Analysis 39: 336-356.

Yongmei, L., and T. Junmei. 2004. "Fractal Dimension of a Transportation Network and its Relationship with Urban Growth: A Study of the Dallas-Fort Worth Area." Environment and Planning B 31: 895-911.

Zipf, G. K. 1949. Human Behaviour and the Principle of Least Effort: An Introduction to Human Ecology. Cambridge, MA: Addison-Wesley. 